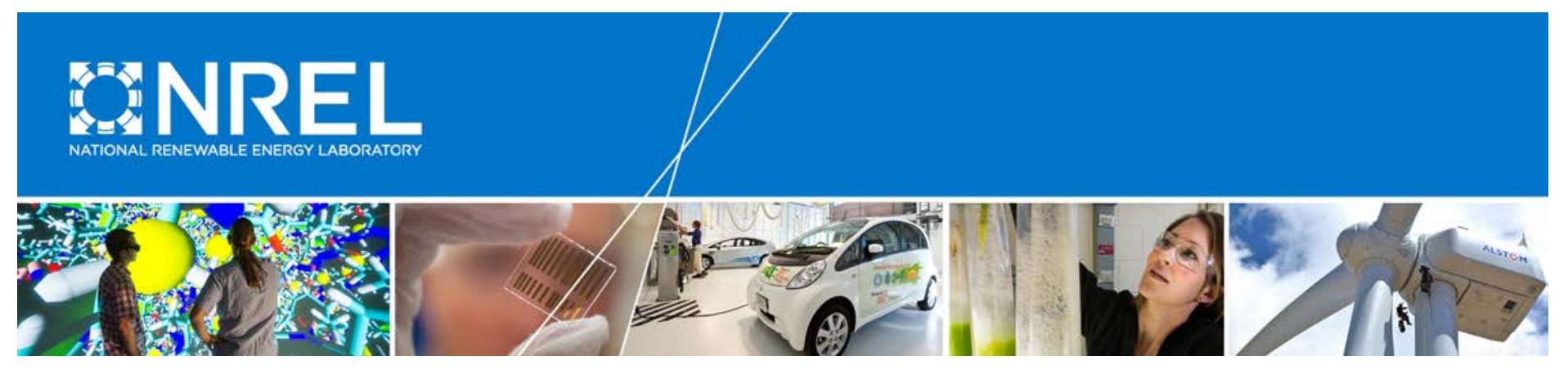

\title{
Modeling Stationary Lithium-Ion Batteries for Optimization and Predictive Control
}

\section{Preprint}

Emma Raszmann University of Pittsburgh

Kyri Baker, Ying Shi, and Dane Christensen National Renewable Energy Laboratory

To be presented at the IEEE Power and Energy Conference Champaign, Illinois

February 23-24, 2017

(C) 2017 IEEE. Personal use of this material is permitted. Permission from IEEE must be obtained for all other uses, in any current or future media, including reprinting/republishing this material for advertising or promotional purposes, creating new collective works, for resale or redistribution to servers or lists, or reuse of any copyrighted component of this work in other works.

NREL is a national laboratory of the U.S. Department of Energy Office of Energy Efficiency \& Renewable Energy Operated by the Alliance for Sustainable Energy, LLC

This report is available at no cost from the National Renewable Energy Laboratory (NREL) at www.nrel.gov/publications.

\section{Conference Paper}

NREL/CP-5D00-67809

February 2017 


\section{NOTICE}

The submitted manuscript has been offered by an employee of the Alliance for Sustainable Energy, LLC (Alliance), a contractor of the US Government under Contract No. DE-AC36-08GO28308. Accordingly, the US Government and Alliance retain a nonexclusive royalty-free license to publish or reproduce the published form of this contribution, or allow others to do so, for US Government purposes.

This report was prepared as an account of work sponsored by an agency of the United States government. Neither the United States government nor any agency thereof, nor any of their employees, makes any warranty, express or implied, or assumes any legal liability or responsibility for the accuracy, completeness, or usefulness of any information, apparatus, product, or process disclosed, or represents that its use would not infringe privately owned rights. Reference herein to any specific commercial product, process, or service by trade name, trademark, manufacturer, or otherwise does not necessarily constitute or imply its endorsement, recommendation, or favoring by the United States government or any agency thereof. The views and opinions of authors expressed herein do not necessarily state or reflect those of the United States government or any agency thereof.

This report is available at no cost from the National Renewable Energy Laboratory (NREL) at www.nrel.gov/publications.

Available electronically at SciTech Connect http:/www.osti.gov/scitech

Available for a processing fee to U.S. Department of Energy and its contractors, in paper, from:

U.S. Department of Energy

Office of Scientific and Technical Information

P.O. Box 62

Oak Ridge, TN 37831-0062

OSTI http://www.osti.gov

Phone: 865.576.8401

Fax: 865.576.5728

Email: reports@osti.gov

Available for sale to the public, in paper, from:

U.S. Department of Commerce

National Technical Information Service

5301 Shawnee Road

Alexandria, VA 22312

NTIS http://www.ntis.gov

Phone: 800.553 .6847 or 703.605 .6000

Fax: 703.605.6900

Email: orders@ntis.gov 


\title{
Modeling Stationary Lithium-Ion Batteries for Optimization and Predictive Control
}

\author{
Emma Raszmann*, Kyri Baker ${ }^{\dagger}$, Ying Shi ${ }^{\dagger}$ and Dane Christensen ${ }^{\dagger}$ \\ * Swanson School of Engineering \\ University of Pittsburgh \\ 3700 O'Hara Street \\ Pittsburgh, Pennsylvania 15213 \\ Email: emma.raszmann@pitt.edu \\ $\dagger$ National Renewable Energy Laboratory \\ 15013 Denver West Pkwy \\ Golden, Colorado 80401
}

\begin{abstract}
Accurately modeling stationary battery storage behavior is crucial to pursuing cost-effective distributed energy resource opportunities. In this paper, a lithium-ion battery model was derived for building-integrated battery use cases. The proposed battery model aims to balance speed and accuracy when modeling battery behavior for real-time predictive control and optimization. To achieve these goals, a mixed modeling approach incorporates regression fits to experimental data and an equivalent circuit to model battery behavior.

The proposed battery model is validated through comparison to manufacturer data. Additionally, a dynamic test case demonstrates the effects of using regression models to represent cycling losses and capacity fading. A proof-of-concept optimization test case with time-of-use pricing is performed to demonstrate how the battery model could be included in an optimization framework.
\end{abstract}

Index Terms-Energy Storage, Batteries, Lithium-Ion, Modeling, Analytical Models, System Integration, Buildings, Optimization.

\section{INTRODUCTION}

Stationary battery storage systems have the potential to provide backup power during outages, reduce electricity costs, and support more integration of sustainable energy sources. Due to these benefits, home battery technologies have been gaining more attention from consumers. In addition, stationary batteries are being sought to overcome grid-integration challenges, including difficulties in economic management by utilities, infrastructure management and grid instabilities. We expect that batteries will be used in conjunction with controllable, dynamic building loads to increase the adoption of renewable energy as well as to manage building energy costs and grid infrastructure risks.

Due to the high cost of battery storage, it is important to optimize how batteries are used to maximize return on investment. We set out to develop a battery model for realtime simulations which can be applied to predictive control scenarios. Dynamic control of loads can be used in parallel to study hybrid load shifting for electricity optimization in timeof-use pricing, which has the potential to mutually benefit both utilities and end users.

Although battery models for electric vehicle and large-scale grid applications are prominent in the literature, the modeling of stationary storage with a particular application to control and optimization has not been as widely studied. Use within an optimization solver motivates a computationally efficient battery model with appropriate fidelity. This paper describes an approach for state-of-charge (SOC) and lifetime estimation of a battery while considering simulation efficiency. Section II of this paper summarizes literature on battery modeling applications and approaches. Section III explains the proposed modeling approach used in this paper, including modeling assumptions for parameters such as battery internal resistance, SOC operation range, and capacity fading. In Section IV, the methodology for validating the battery model against other models is explained. Section V shows how the specific battery model presented in this paper was converted to units of power and energy. In Section VI, an example of how to apply this stationary battery model to avoid incurring additional electricity costs in an optimization scheme is presented. Finally, in Section VII, performance comparisons among battery models are discussed, and potential directions for future work in this area are given.

\section{BACKGROUND}

\section{A. Lithium-Ion Batteries}

Lithium-ion batteries are commonly used in portable electronics, but recently they have gained popularity in larger scale applications such as grid-tied systems and electric vehicles. When selecting a battery for residential applications, lifetime and maintenance should be considered. Lithium-ion battery packs have a higher round-trip efficiency than many other alternatives, which can lead to a longer lifetime compared to other battery types such as lead acid [1]. Other benefits of 
lithium-ion batteries include lower volume, weight, temperature sensitivity, and maintenance.

\section{B. Battery Modeling Applications}

1) Grid-Scale: Computational efficiency is a priority for grid-scale simulations of battery models because of the volume of loads to simulate. Equation (1) shows a common grid-scale battery model for calculating the voltage of a battery based on charge and discharge efficiency [2], [3].

$$
\begin{aligned}
E_{B}(t) & =E(0)+\sum_{t=1}^{T} E(t) \\
E(t) & =P_{B}(t) \Delta t \\
E_{\min } & \leqslant E(t) \leqslant E_{\text {max }}
\end{aligned}
$$

where $E(0)$ represents the initial battery energy, $E(t)$ represents the battery energy at time $t, \Delta t$ represents the time in between control decisions, $T$ represents the length of time that the battery is used, and $P_{B}(t)$ represents the instantaneous power of the battery at time $t . E_{\min }$ and $E_{\max }$ represent the minimum and maximum stored energy limits, respectively, based on the SOC operation range for the battery [2], [3].

Although (1) is simple to compute because it is a first-order linear equation, this representation omits the consideration of the effects of temperature on internal resistance and capacity fading. Accurate battery lifetime and SOC prediction were the motivation behind the battery model described in this paper.

2) Electric Vehicles (EVs): Battery storage plays a significant role in the overall efficiency of EVs. Models for EVs have a greater variability in charging and discharging cycles than grid-scale battery storage due to the effects of acceleration and deceleration; therefore, a more detailed model than (1) is desirable for EVs.

Voltage-current equivalent circuit models describe how the terminal voltage of the battery changes with current. Shepherd's model [4] is a well-known voltage-current model for constant current discharge, as shown in (2).

$$
V_{b a t t}(t)=E_{0}-K \frac{Q}{Q-i t} i(t)-R_{O} * i(t)
$$

where $E_{0}$ is the constant battery voltage $(V), K$ is polarization constant $(V / A h), Q$ is battery capacity $(A h), V_{\text {batt }}(t)$ is terminal battery voltage $(V)$ at time $t$, it is discharged capacity $(A h)$, and, $i(t)$ is the dynamic current $(A)$ at time $t$. These terms will be explained in more detail later on in the paper.

To derive battery parameters for Shepherd's model, the discharge curve from the battery manufacturer can be used [4]. An idealized discharge curve is shown in Figure 1. The three points shown on the discharge curve in Figure 1 can be used to determine the constant parameters $K, R_{O}$, and $E_{0}$ in (1).

Shepherd's battery model is often modified to expose exponential battery behavior in more detail [5], [6]. The charge and discharge equations for the modified Shepherd's model are shown in (3) and (4), respectively [5].

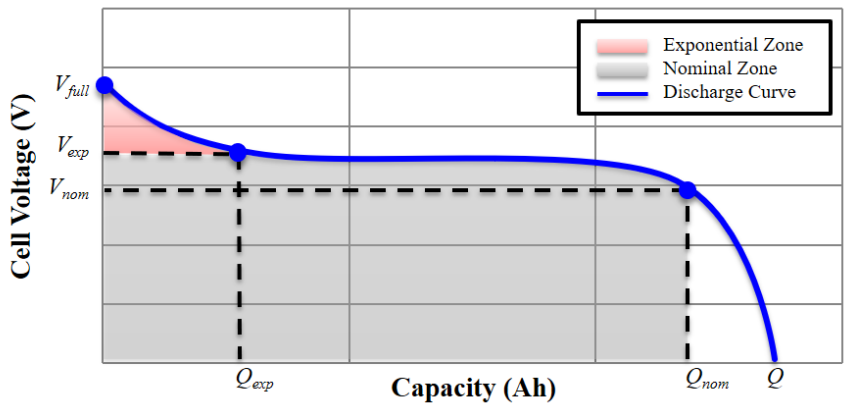

Fig. 1. Example of idealized battery discharge curve.

$$
\begin{array}{r}
V_{\text {batt }}(t)=E_{0}-K \frac{Q}{i t-0.1 Q}\left(i(t)+i^{*}\right)-R_{O} * i(t) \\
+A e^{-B * i t} \\
V_{\text {batt }}(t)=E_{0}-K \frac{Q}{Q-i t}\left(i(t)+i^{*}\right)-R_{O} * i(t) \\
+A e^{-B * i t}
\end{array}
$$

where A represents the amplitude in the exponential zone $(V)$, B represents the time constant inverse in the exponential zone $\left(A h^{-1}\right)$, and $i^{*}$ represents the current filtered through polarization resistance $(A)$. Similar to (1), parameters A and $B$ can also be determined from the manufacturing discharge curve.

Grid-scale and EV applications are only two of many ways to use battery storage. These applications were discussed to provide examples of general approaches to battery modeling. As shown in discussing these two applications, the selected model depends heavily on the given application. In this paper, we focus on the development of a residential stationary battery model.

\section{Equivalent Circuit Models}

A wide range of battery modeling approaches depend on what details are desired from the model, including experimental, electrochemical, and electric circuit-based models [5]. Equivalent circuit models are simple and can represent steadystate and dynamic battery behavior [5], [6]. An equivalent circuit approach is preferable for modeling stationary battery behavior for predictive control because of its faster simulation time [7]. With higher order models, the optimization problem is rendered nonconvex, and it may not be solved in an efficient manner. This is particularly important for real-time optimization and control on short timescales.

The $R_{\text {int }}$ equivalent circuit model contains a constant voltage source in series with a resistor, as shown in Figure 2 [8]. In Figure 2, $i(t)$ represents the battery's internal dynamic current $(A), R_{O}$ represents the battery's internal ohmic resistance (ohms), $V_{\text {batt }}$ represents the terminal output voltage of the battery, and $V_{O C}$ represents the applied input voltage to the 


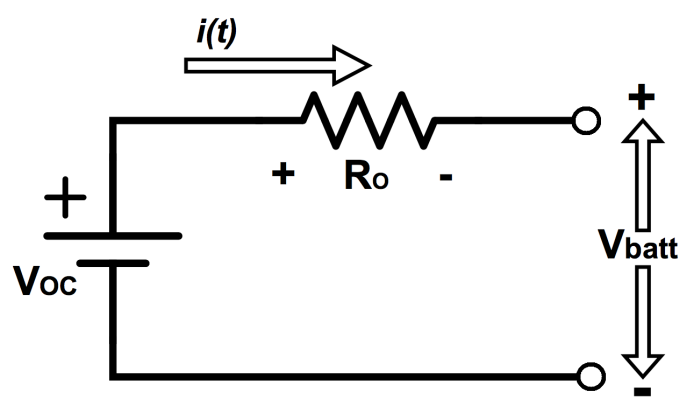

Fig. 2. Circuit diagram of $R_{\text {int }}$ equivalent circuit model.

battery. The terminal voltage output for the $R_{\text {int }}$ model is shown below:

$$
V_{\text {batt }}(t)=V_{O C}-R_{O} * i(t)
$$

This model does not include the battery SOC directly; however, the battery SOC can be represented as a function of the open-circuit voltage [5]. Other equivalent circuits in the literature model the effects of polarization in the battery using RC parallel circuits. Although these models may be more accurate than the $R_{\text {int }}$ model, the differential equations that represent capacitors require greater simulation times. This cost outweighed the benefits for our purpose, so we selected the $R_{\text {int }}$ equivalent circuit.

\section{Proposed Modeling Approach}

\section{A. Voltage-Current Equations}

Regression fits to manufacturing data and an equivalent circuit were used to represent the charging and discharging behavior of the battery. The proposed voltage-current equations are a mix between Shepherd's model in (2) and the modified versions of Shepherd's model in (3) and (4). Parameters A and B in (3) and (4) are derived from the points found in the exponential zone of the idealized discharge curve shown in Figure 1. The particular discharge curve used in this paper is shown in Figure 3.

As shown in Figure 3, batteries can be discharged at different rates of current. C-rates refer to the rate of constant current that will cause the battery to discharge in a certain amount of hours. For example, a $0.2 \mathrm{C}$ rate represents the rate of current required to drain the battery in five hours, and a $1 \mathrm{C}$ rate represents the rate of current required to drain the battery in one hour.

To calculate parameters for the voltage-current relationships in this model, three points from Figure 3 were selected based on the shape of the idealized curve shown in Figure 1. The three pairs of voltage and capacity values for the $1 \mathrm{C}$ rate are shown below:

$$
\begin{aligned}
\left(Q_{\text {full }}, V_{\text {full }}\right) & =(31 \mathrm{Ah}, 4.18 \mathrm{~V}) \\
\left(Q_{\text {exp }}, V_{\text {exp }}\right) & =(22.5 \mathrm{Ah}, 3.515 \mathrm{~V}) \\
\left(Q_{\text {nom }}, V_{\text {nom }}\right) & =(29.2 \mathrm{Ah}, 3.35 \mathrm{~V})
\end{aligned}
$$

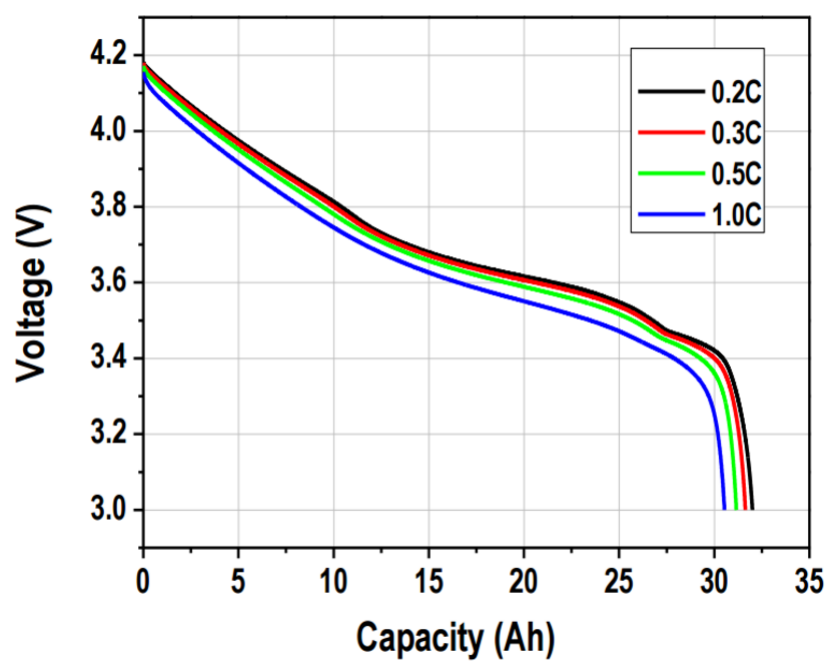

Fig. 3. Discharge curve for stationary battery at various C-rates.

The exponential zone of the discharge curve shown in Figure 3 is linear compared to the idealized curve shown in Figure 1. In this case, $V_{\text {exp }}$ was estimated as the voltage at approximately $15 \%$ of the full capacity of the battery.

Next, the following battery parameter values were calculated based on these three pairs of points from the discharge curve:

1) Exponential Voltage Amplitude Constant (A, in units of $V$ ): The amplitude of the exponential region, or $\mathrm{A}$, is calculated with (6) [6].

2) Time Constant Inverse $\left(B\right.$, in units of $\left.A h^{-1}\right)$ : The charge at the end of the exponential zone of the battery's discharge curve, or B, is calculated using (7) [6]. The scalar value of 2.3 in (7) was used to improve the fit to the particular battery data shown in Figure 3.

3) Polarization Constant ( $K$, in units of $V / A h)$ : The polarization constant, $K$, is calculated using $V_{\text {full }}$ and the end of the nominal zone of the discharge curve, as shown in (8) [6]. Similar to (7), the scalar value of 0.065 in (8) was used to improve the fit to the battery discussed in this paper.

4) Internal Resistance ( $R$, in units of $\Omega$ ): The internal resistance of the battery at steady-state current is represented by R, as shown in (9) [6], where $v$ represents the efficiency of the battery, and $i$ represents the nominal current used to test the steady-state discharge of the battery.

5) Battery Constant Voltage ( $E_{0}$, in units of $V$ ): The battery constant voltage represents the value when the battery is close to completely discharged and no current is flowing. The relationship used to calculate $E_{0}$ is shown in (10) [6].

The resulting voltage-current equation for steady-state discharge representation of the battery is shown in (11) [6], where $i$ represents a constant current input at a given Crate. The dynamic charging and discharging relationships of the battery model are shown in (12) and (13), respectively [5]. The term $i^{*}$, which represents the filtered current through polarization resistance in (3) and (4), was replaced with $i(t)$ because polarization resistance effects were not considered for the proposed model. 


$$
\begin{aligned}
& A=V_{\text {full }}-V_{\text {exp }} \\
& B=\frac{2.3}{Q_{\text {exp }}} \\
& K=0.065 *\left[V_{\text {full }}-V_{\text {nom }}+\right. \\
& \left.A\left(e^{-B * Q_{\text {nom }}}-1\right)\right] * \frac{Q_{f u l l}-Q_{\text {nom }}}{Q_{\text {nom }}} \\
& R=V_{\text {nom }}\left(\frac{1-v}{0.2 * Q_{\text {nom }}}\right) \\
& E_{0}=V_{f u l l}+K+R * i-A \\
& V_{b a t t}(t)=E_{0}-K \frac{Q}{i t-0.1 Q}+A e^{-B * i t}-R_{O} * i \\
& V_{\text {batt }}(t)=E_{0}-K \frac{Q}{i t-0.1 Q}(i t+i(t))+A e^{-B * i t}-R_{O} * i(t) \\
& V_{\text {batt }}(t)=E_{0}-K \frac{Q}{Q-i t}(i t+i(t))+A e^{-B * i t}-R_{O} * i(t)
\end{aligned}
$$

\section{B. Assumptions for Model Parameters}

1) Internal Resistance: In the proposed battery model, the internal resistance of the battery was assumed to be a function of both the temperature and SOC. With data provided by the manufacturer of the lithium-ion battery, a multiple linear regression relationship was determined to represent $R_{O}$. To prevent over-fitting the data, a first-order regression fit was selected, as shown below:

$$
R_{O}=c_{1} * T+c_{2} * i t+c_{3}
$$

where $c_{1}, c_{2}$, and $c_{3}$ are constants determined by multiple linear regression, $T$ represents temperature, and it is the battery's discharged capacity $(A h)$.

2) Capacity Fading: Similar to how the internal resistance of the battery was modeled, a multiple linear regression relationship was formed for $Q$, the battery capacity, as a function of the temperature and the total number of cycles of the battery. The regression model was kept as a first-order linear equation to prevent over-fitting the data and to optimize the simulation time of the overall model.

3) State-of-Charge Range: Due to the degradation effects of the battery, such as polarization and capacity fading, the battery was limited to operating within the linear region of its nominal discharge curve, as shown in Figure 3. The discharge curve's linear region was estimated to be between $20 \%$ and $85 \%$ of the full capacity of each battery cell, based on Figure 1 ; therefore, the model was limited to charge and discharge between $20 \%$ and $85 \%$ SOC.
4) Temperature Range: In this paper, temperature refers to the ambient temperature inside the cabinet for the stationary battery. The temperature range for battery operation can typically be found in the battery's datasheet. The range used in this paper was between $-10^{\circ} \mathrm{C}$ and $45^{\circ} \mathrm{C}$. Operating outside of the range tested and provided by the manufacturer can significantly degrade the capacity of the battery over time.

To summarize these considerations for model parameters, Figure 4 shows a flow diagram of the order of operations to determine the dynamic behavior of the battery. At each iteration in the dynamic battery model simulation, capacity fading and internal resistance effects were updated with the regression models, as previously discussed.

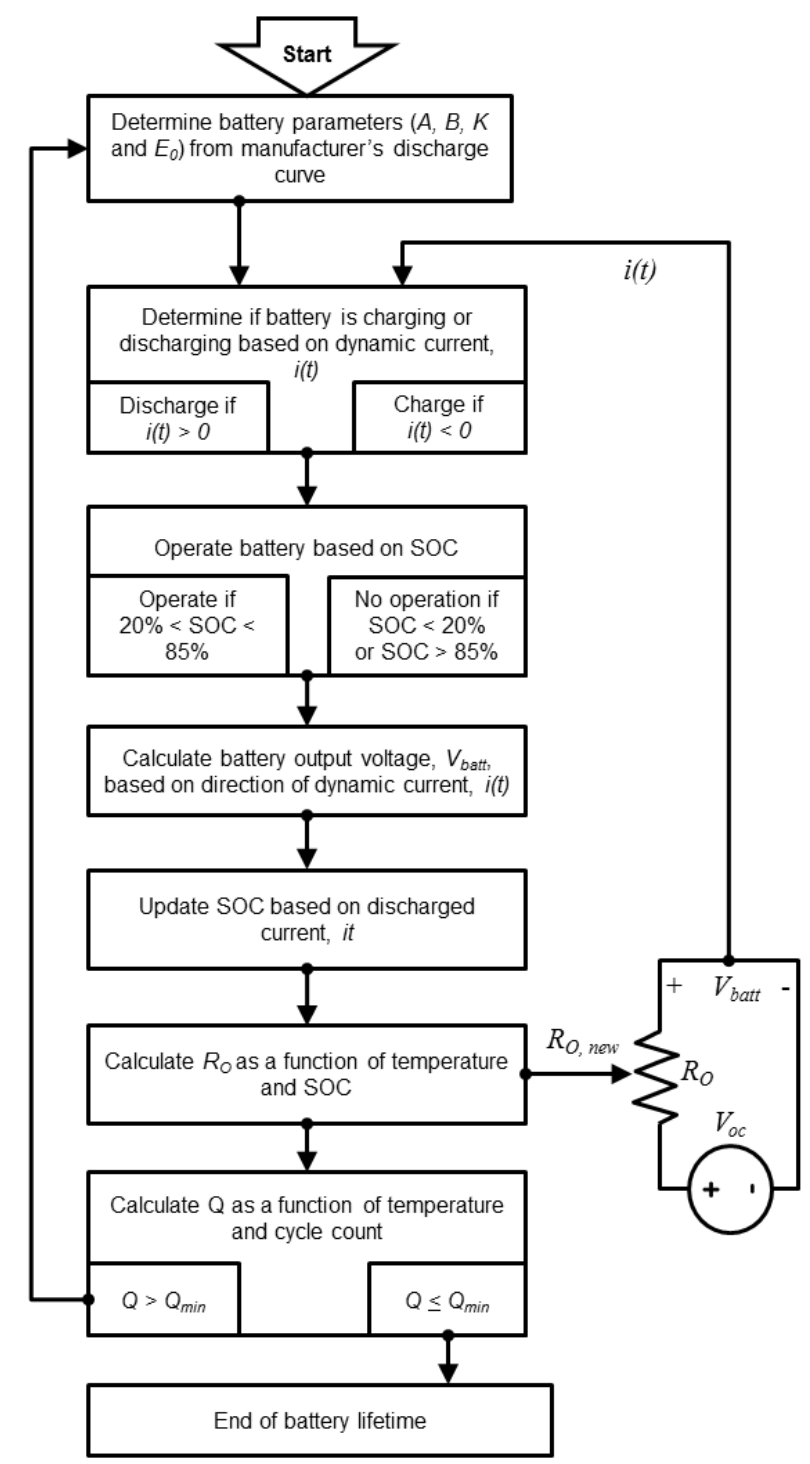

Fig. 4. Flow diagram explaining how to calculate the proposed battery model output, $V_{\text {batt }}$.

The battery output voltage was updated at each time step using (12) and (13). The SOC was estimated with (15) and 
(16), as shown below:

$$
\begin{gathered}
\operatorname{SOC}(t)=\operatorname{SOC}(t-1)+\frac{i(t) \cdot \Delta t}{Q}, \text { if } Q \text { is in } A h \\
\operatorname{SOC}(t)=\operatorname{SOC}(t-1)+\frac{P(t) \cdot \Delta t}{Q}, \text { if } Q \text { is in } W h
\end{gathered}
$$

where $\Delta t$ indicates the amount of time between timesteps $t-1$ and $t$. The battery behavior is continually updated until the remaining capacity is no longer usable, or when $Q \leq Q_{\min }$, where $Q_{\min }$ represents the minimum allowable battery capacity. In the proposed model, $Q_{\min }$ was set to the capacity at $20 \%$ SOC on the discharge curve shown in Figure 3.

\section{Model Validation}

\section{A. Steady-State Behavior}

To validate the battery model accuracy against the manufacturer's discharge curve, a steady-state current was used as an input. The discharge curve for the battery used in steadystate validation is shown in Figure 3. The simulation results of the steady-state validation were superimposed on the original manufacturing data, as shown in Figure 5.

In Figure 5, the shaded region represents the operational SOC range of the battery model. Thus, the accuracy of the fit between the simulated curve and manufacturing curve is most crucial within the allowable SOC range for the battery, which was selected to be between 20\% (6.2 $A h)$ and 85\% $(26.35 A h)$. As shown in Figure 5, the error between curves within the chosen allowable SOC range for the battery is negligible. Upon verifying the fit between the experimental battery manufacturing data and the simulated discharge curve, the steady-state simulation parameters are used to predict the dynamic behavior of the battery.

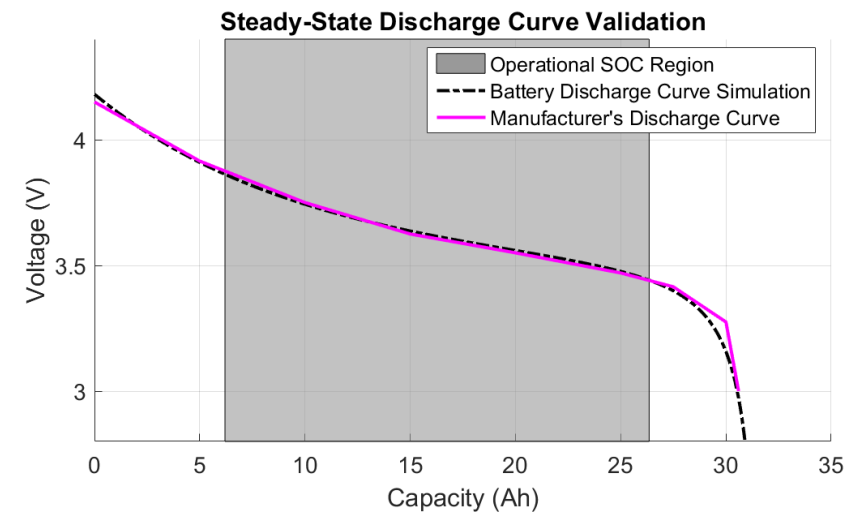

Fig. 5. Steady-state discharge curve output plotted against the manufacturer's data at a rate of $1 \mathrm{C}$ as an example.

\section{B. Dynamic Behavior}

To evaluate the model discussed in this paper, dynamic battery behavior was validated with different manufacturing data but the same battery type. Temperature effects were not considered in this test case, but could be included using the regression model procedure described in Section III. To test the proposed battery model with a dynamic test case, results for a $2.3 \mathrm{~V}, 3.3 \mathrm{Ah}$ lithium-ion EV battery model were used for comparison, as described in [5]. Obtained from the battery's discharge curve, this battery had the following steady-state parameters [5]: $E_{0}=3.366 \mathrm{~V}, K=0.0076 \mathrm{~V} / \mathrm{Ah}, \mathrm{A}=0.26422$ $V, \mathrm{~B}=26.5487 A h^{-1}$, and $R=0.01 \Omega$. Using these parameters, the dynamic test case from [5] was also simulated with the model proposed in this paper for comparison, as shown in Figure 6.
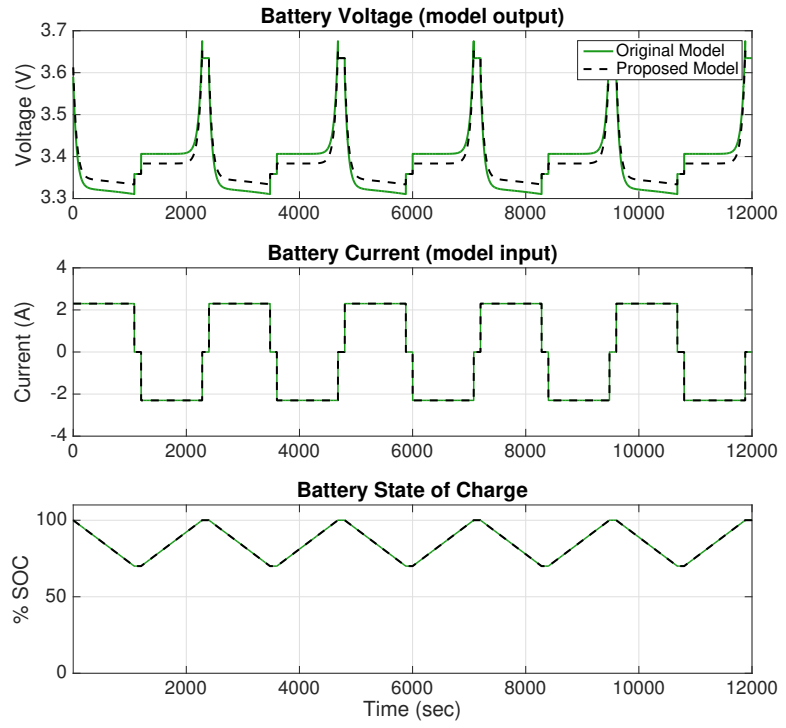

Fig. 6. Dynamic test case for a $2.3 \mathrm{~V}, 3.3 \mathrm{Ah}$ lithium-ion battery.

The test case shown in Figure 6 was simulated to represent battery operation over the span of 200 minutes. For this test case, because the current input in Figure 6 causes a selfsustaining cycling of the battery, the $V_{\text {batt }}$ output shows a repeating pattern without any degradation effects to the output during this period of time. The regression model for the internal resistance of the battery contributed the most to the error between the original model and the proposed model shown in Figure 6.

\section{Expressing Stationary Battery Model in the POWER AND ENERGY DOMAIN}

In the field of electrochemistry, it is conventional to model batteries in terms of current and voltage. However, in modeling a stationary battery for demand-side management scenarios in power and energy systems, it is more useful to express the battery model in terms of power and energy. The type of experimental data that is provided about a given battery can vary depending on the battery manufacturer. In the example described in this paper, the battery manufacturer published both C-rate and CP-rate steady-state discharge curves for the same battery. Similar to C-rate terminology previously explained in Section III, CP-rates refer to the rate of constant power that will cause the battery to discharge in a certain 
amount of hours. To compare between existing battery models, the C-rate curve was used to first develop the model described in this paper.

To represent this model in the power and energy domain, voltage and current values derived from Figure 3 were used to convert the proposed model to units of $W h$ for the particular battery discussed in this paper. Figure 7 shows a CP-rate plot for the battery, where lines with a negative slope denote the discharge curves and lines with a positive slope denote the charging curves. As shown in Figure 7, voltage values obtained from the $y$-axis of the C-rate plot in Figure 3 with approximations of energy values as the $\mathrm{X}$-axis were superimposed on top of the original CP-rate curve. To calculate energy $(W h)$ points from the original capacity $(A h)$ data, the Newton-Raphson estimation method was used, as shown in (17) below:

$$
f(t)=f(t-1)+x(t) *[y(t)-y(t-1)]
$$

where $\mathrm{f}(\mathrm{t})$ represents energy in $W h, \mathrm{x}(\mathrm{t})$ represents voltage, and $\mathrm{y}(\mathrm{t})$ represents capacity in $A h$. Approximations for $\mathrm{CP}$ rate curves were plotted against the manufacturer's original CP-rate data, as shown in Figure 7.

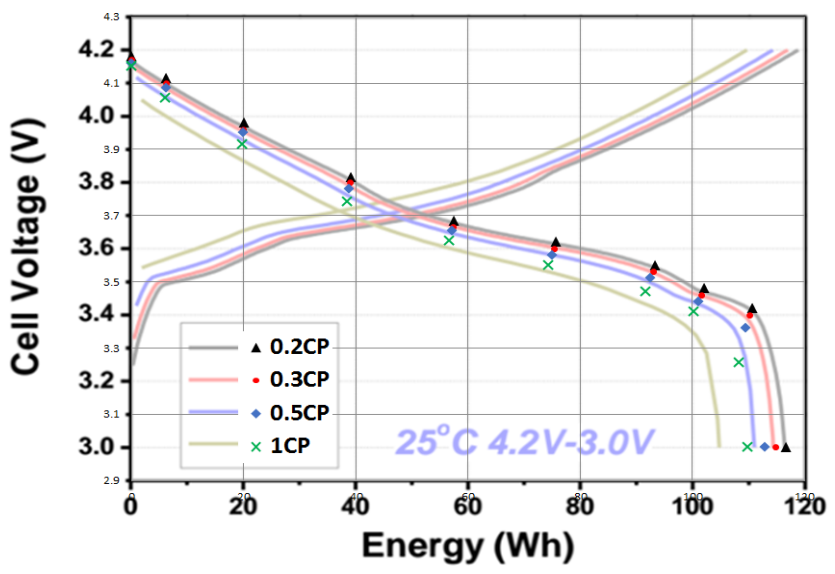

Fig. 7. Validation curve for converting original data from units of $A h$ to $W h$. The lines are the original battery manufacturer's CP-rate curves, and the points are approximations to each curve based on the approach described in Section V of this paper.

Although the approximations shown in Figure 7 show a margin of error between the actual manufacturing curves, this is likely due to how these approximations assume linear losses between each different discharge rate. For this particular battery, the CP-rate curve approximations shown in Figure 7 validate that the $\mathrm{C}$-rate discharge curve in Figure 3 can be transformed from units of $A h$ to $W h$ to generally represent the battery's behavior in the power and energy domain, which is useful for many applications in power and energy systems optimization and control.

We further note that greater error appears to be incurred at higher discharge rates, supporting the above finding that this error derives from the representation of internal resistance. At higher currents, $i^{2} R$ losses have a greater effect on the model output. For our use case, the inverter discharging rate was limited to a maximum of $0.5 \mathrm{CP}$, so we have determined the error to be acceptable.

\section{Optimization Test Case}

To demonstrate an example of where the aforementioned battery model in the power and energy domain would fit into an optimization scheme, a simple test case was performed. In the test case, the objective was to minimize the cost of electricity drawn from the grid while maintaining power balance between demand and generation in a residential building. It is assumed that both load and solar irradiance predictions were perfectly known (i.e., forecasting errors are not included here). The optimization was performed every thirty minutes, with the optimization variables being power charged or discharged from the battery and power drawn from the grid. As an example, a time-of-use (TOU) electricity price, which is twice as expensive between the hours of 1:00 PM and 2:30 PM, incentivizes the optimization to precharge the battery before the price increase and utilize energy from the battery during the peak price, as seen in Figure 8 . The convention taken in the figure is to include power "consumption" by the home as a positive value, thus the charging of the battery in Figure 8 is plotted as positive.

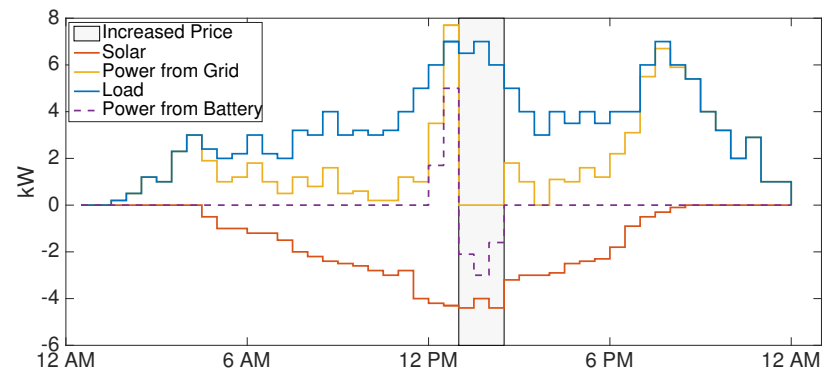

Fig. 8. Total power consumption and generation over the course of a day with 30-minute control periods.

In this case, the homeowner saved $14 \%$ on their electricity bill due to avoiding drawing power from the grid during the time when the electricity price doubled. Figure 9 shows the battery state of charge (with an initial, minimum state of charge set to $25 \%$ ) over the course of a day resulting from the charging/discharging pattern determined by the optimization scheme.

In contrast to Figure 8, battery charging is denoted with a negative value to be consistent with the convention in energy storage modeling. In this simple test case, the battery is utilized to avoid incurring higher costs during the TOU pricing period by looking forward and anticipating the increase in price. However, as discussed previously, the optimization of battery controls has a wide range of applications in the field of predictive control, from load-shifting, to supplying the 

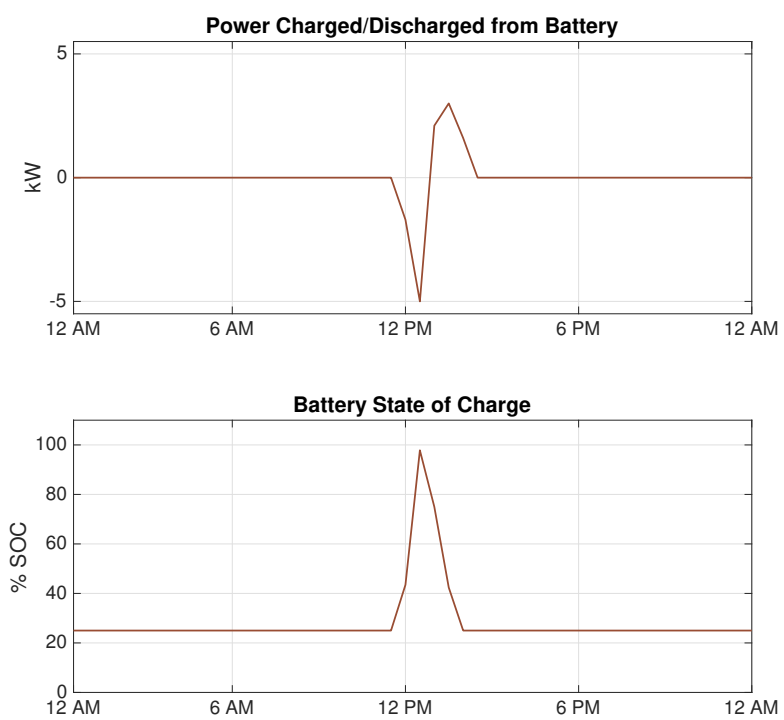

Fig. 9. Battery model behavior during the optimization scenario.

load during demand response events, to balancing renewable fluctuations.

\section{CONCLUSIONS}

This paper demonstrates how to model SOC effects on internal resistance and capacity fading using multiple linear regression models that are appropriate for optimization and predictive control. Section IV validates this process by comparing it to the dynamic results for an EV model from the literature. By demonstrating a small percentage error between the original and new battery model voltage outputs, it is shown that dynamically modeling internal resistance and capacity fading with regression models did not cause over-fitting of the data. A small test case demonstrating how the use of battery storage could avoid drawing power from the grid during peak pricing times was performed.

To further validate the battery model proposed in this paper, future steps involve experimentally measuring the output of a battery and comparing it to the predicted output of the dynamic simulation. Temperature effects were not considered in the dynamic simulations shown in Section IV of this paper, but in future work, the impact of temperature dependencies on internal resistance and capacity fading should be studied. In addition to comparing the modeling prediction to experimental measurements, the model should be tested with a more complex optimization scenario to demonstrate its fidelity and robustness.

\section{ACKNOWLEDGMENT}

This work was supported by the U.S. Department of Energy under Contract No. DE-AC36-08GO28308. Funding provided by the Bonneville Power Administration, the U.S. Department of Energy, Energy Efficiency and Renewable Energy Office (EERE) Building Technologies Program, and the U.S. Department of Energy, Office of Science, Office of Workforce Development for Teachers and Scientists (WDTS) under the Science Undergraduate Laboratory Internships Program (SULI). Cost share on the project is provided by Robert Bosch, N.A. and ESCRYPT. Battery data courtesy of Eguana, Inc. The U.S. Government retains and the publisher, by accepting the article for publication, acknowledges that the U.S. Government retains a nonexclusive, paid-up, irrevocable, worldwide license to publish or reproduce the published form of this work, or allow others to do so, for U.S. Government purposes.

\section{REFERENCES}

[1] A. Technologies, "A comparison of lead acid to lithium-ion in stationary storage applications," 2012, [Battery Power Online, http://www.batterypoweronline.com/main/wp-content/uploads/2012/ 07/Lead-acid-white-paper.pdf, accessed August 12, 2016].

[2] K. Baker, J. Guo, G. Hug, and X. Li, "Distributed MPC for efficient coordination of storage and renewable energy sources across control areas," IEEE Transactions on Smart Grid, vol. 7, pp. 992-1001, March 2016.

[3] C. Zhao, S. Dong, F. Li, and Y. Song, "Optimal home energy management system with mixed types of loads," CSEE Journal of Power and Energy Systems, vol. 1, no. 4, pp. 29-37, December 2015.

[4] S. Li and B. Ke, "Study of battery modeling using mathematical and circuit oriented approaches," 2011 IEEE Power and Energy Society General Meeting, pp. 1-8, 2011.

[5] O. Tremblay and L. A. Dessaint, "Experimental validation of a battery dynamic model for EV applications," World Electric Vehicle Journal, vol. 3, pp. 289-298, May 2009.

[6] O. Tremblay, L. A. Dessaint, and A. I. Dekkiche, "A generic battery model for the dynamic simulation of hybrid electric vehicles," 2007 IEEE Vehicle Power and Propulsion Conference, pp. 284-289, September 2007.

[7] R. Rao, S. Vrudhula, and D. N. Rakhmatov, "Battery modeling for energy aware system design," Computer, vol. 36, no. 12, pp. 77-87, December 2003.

[8] H. He, R. Xiong, H. Guo, and S. Li, "Comparison study on the battery models used for the energy management of batteries in electric vehicles," Energy Conversion and Management, vol. 64, pp. 113-121, December 2012. 